CERN-TH/2001-057

KEK-TH-749

February 2001

hep-ph/yymmdd

\title{
Neutrino masses, muon $g-2$, and lepton-flavour violation in the supersymmetric see-saw model
}

\author{
Junji Hisano $^{(a, b)}$ and Kazuhiro Tobe ${ }^{(b)}$ \\ (a) Theory Group, KEK, Oho 1-1, Tsukuba, Ibaraki 305-0801, Japan \\ (b) Theory Division, CERN, 1211 Geneva 23, Switzerland
}

\begin{abstract}
In the light of the recent muon $\left(g_{\mu}-2\right)$ result by the E821 experiment at the Brookhaven National Laboratory, we study the event rates of the charged leptonflavour-violating (LFV) processes in the supersymmetric standard model (SUSY SM) with the heavy right-handed neutrinos (SUSY see-saw model). Since the left-handed sleptons get the LFV masses via the neutrino Yukawa interaction in this model, the event rate of $\mu \rightarrow e \gamma$ and the SUSY-SM correction to $\left(g_{\mu}-2\right) / 2$ $\left(\delta a_{\mu}^{\mathrm{SUSY}}\right)$ are strongly correlated. When the left-handed sleptons have a LFV mass between the first and second generations $\left(\left(m_{\tilde{L}}^{2}\right)_{12}\right)$ in the mass matrix, it should be suppressed by $\sim 10^{-3}\left(10^{-9} / \delta a_{\mu}^{\mathrm{SUSY}}\right)$ compared with the diagonal components $\left(m_{\text {SUSY }}^{2}\right)$, from the current experimental bound on $\mu \rightarrow e \gamma$. The recent $\left(g_{\mu}-2\right)$ result indicates $\delta a_{\mu}^{\mathrm{SUSY}} \sim 10^{-9}$. The future charged LFV experiments could cover $\left(m_{\tilde{L}}^{2}\right)_{12} / m_{\text {SUSY }}^{2} \gtrsim 10^{-(5-6)}$. These experiments will give a significant impact on the flavour models and the SUSY-breaking models. In the SUSY see-saw model $\left(m_{\tilde{L}}^{2}\right)_{12}$ is proportional to square of the tau-neutrino Yukawa-coupling constant. In the typical models where the neutrino-oscillation results are explained and the top-quark and tau-neutrino Yukawa couplings are unified at the GUT scale, a large LFV mass of $\left(m_{\tilde{L}}^{2}\right)_{12} / m_{\text {SUSY }}^{2} \gtrsim 10^{-4}$ is generated, and the large LFV event rates are predicted. We impose a so-called no-scale condition for the SUSY-breaking parameters at the GUT scale, which suppresses the FCNC processes, and derive the conservative lower bound on $\mu \rightarrow e \gamma$. The predicted $\operatorname{Br}(\mu \rightarrow e \gamma)$ could be covered at the future LFV experiments.
\end{abstract}


Various neutrino oscillation experiments suggest the existence of lepton-flavour violation (LFV). The atmospheric neutrino result by the superKamiokande experiment is a convincing evidence of the neutrino mixing between $\nu_{\mu}$ and $\nu_{\tau}$ [1]. Solar neutrino experiments have also strongly indicated the mixing between $\nu_{e}$ and $\nu_{\mu}$ or $\nu_{\tau}$ [2]. These results are explained by the tiny but non-zero neutrino masses, and the natural model for the neutrino masses is the see-saw mechanism [3].

It has been discussed that the supersymmetric (SUSY) extension of the see-saw mechanism (SUSY see-saw model) could lead to the charged-LFV processes, such as $\mu \rightarrow e \gamma$ [4, 5, 6, 7, 8. If the SUSY-breaking terms in the supersymmetric standard model (SUSY SM) are generated by the interaction at the gravitational scale or the GUT scale, the Yukawa interaction of the right-handed neutrinos generates the LFV masses for the lefthanded sleptons radiatively, and the LFV masses are a direct source of the charged-LFV processes in the SUSY SM. Especially, in the SUSY models with Yukawa-coupling unification between the top quark and tau neutrino, the event rates of the charged-LFV processes are significantly enhanced, since the LFV masses is proportional to square of the tau-neutrino Yukawa-coupling constant. In many proposed models, this assumption is adopted so that the fermion mass structure, including the neutrino sector, is explained in a unified picture between quarks and leptons, of which a well-known example is the SO(10) GUT. If the left-handed slepton, chargino and neutralino are light, the predicted event rates could be within reach of the near-future experiments.

Recently, the ongoing E821 experiment at the Brookhaven National Laboratory updated the result for the muon anomalous magnetic moment $\left(g_{\mu}-2\right)$ [9], and it is found that $\left(g_{\mu}-2\right)$ is about $2.6 \sigma$ away from the SM prediction as

$$
a_{\mu}(\exp )-a_{\mu}(\mathrm{SM})=43(16) \times 10^{-10}
$$

This suggests that new physics exists around the TeV scale [10], and this result will be further refined after including the data for the 2000 run. The SUSY SM predicts a sizable deviation from the SM prediction for $\left(g_{\mu}-2\right)$ [11, and it is a good candidate to accommodate the $\left(g_{\mu}-2\right)$ result [12]. This comes from the fact that the dominant correction to $\left(g_{\mu}-2\right)$ in the SUSY SM is from a one-loop diagram of chargino and muon sneutrino and it is proportional to $\tan \beta$. This new observation indicates that the chargino, 
the left-handed smuon and the sneutrino are relatively light or that $\tan \beta$ is much larger than 1.

In this situation the LFV processes of muon will be extremely interesting, since the event rates for the LFV processes are enhanced by the light chargino and slepton or large $\tan \beta$. In fact, when the left-handed sleptons have LFV masses, the 1-loop diagrams in $\mu \rightarrow e \gamma$ have the same structure as those in the SUSY-SM correction to $\left(g_{\mu}-2\right)$. As a result, the event rate for $\mu \rightarrow e \gamma$ is approximately proportional to the square of the SUSY-SM correction to $\left(g_{\mu}-2\right)$. The other LFV processes of muon, $\mu \rightarrow 3 e$ and $\mu-e$ conversion in nuclei, are also enhanced since the photon penguin diagram tends to dominate in those processes.

In this letter, in the light of the recent $\left(g_{\mu}-2\right)$ result, we derive the conservative event rates of the charged-LFV processes in the SUSY see-saw models with Yukawa-coupling unification. In order to derive the conservative prediction of the LFV event rates, we consider a so-called no-scale-type SUSY breaking at the GUT scale 15 (or recently called gaugino mediation [16]). This means that all SUSY-breaking scalar masses and A-terms vanish at the GUT scale, and hence the dominant source of SUSY breaking is nonzero gaugino masses. This no-scale-type SUSY breaking is well-known as a suppression mechanism of the SUSY FCNC problem. Since the $\left(g_{\mu}-2\right)$ result gives the upper bounds of the slepton and chargino masses, this limit supplies the conservative lower bound on the LFV event rates. We show that the proposed improvement of limits on the LFV rare muon decay processes and the present indication of anomaly for $\left(g_{\mu}-2\right)$ will provide a significant impact on the flavour models as well as the SUSY-breaking mechanism.

Before starting to discuss the charged-LFV processes in the SUSY SM with the righthanded neutrinos, we show the strong correlation between the event rate of $\mu \rightarrow e \gamma$ and the SUSY-SM correction to $\left(g_{\mu}-2\right)$ when the left-handed sleptons have the LFV masses. These are generated by one-loop diagrams mediated by sleptons, neutralinos and charginos. The effective operators for $\mu \rightarrow e \gamma$ and $\left(g_{\mu}-2\right)$ are

$$
\mathcal{L}_{e f f}=e \frac{m_{l_{j}}}{2} \bar{l}_{i} \sigma_{\mu \nu} F^{\mu \nu}\left(L_{i j} P_{L}+R_{i j} P_{R}\right) l_{j}
$$

\footnotetext{
1 In Ref. [13] the relation between $\left(g_{\mu}-2\right)$ and the charged LFV processes is presented in nonsupersymmetric models to generate finite neutrino masses. Also, in Ref. [1] the relation is discussed in the $Z^{\prime}$ model with the flavor-dependent interaction.
} 
where $m_{l_{i}}$ is a mass of the charged lepton $l_{i}$, e and $F^{\mu \nu}$ are the QED coupling constant and field strength, and $P_{R / L}=\left(1 \pm \gamma_{5}\right) / 2$. The coefficients $L_{i j}$ and $R_{i j}$ are functions of the SUSY-particle masses and mixings. From these operators (2), the branching ratio for $\mu \rightarrow e \gamma$ is given by

$$
\operatorname{Br}(\mu \rightarrow e \gamma)=\frac{48 \pi^{3} \alpha}{G_{F}^{2}}\left(\left|L_{12}\right|^{2}+\left|R_{12}\right|^{2}\right),
$$

and the SUSY-SM correction to $a_{\mu}\left(\equiv\left(g_{\mu}-2\right) / 2\right)$ is

$$
\delta a_{\mu}^{\mathrm{SUSY}}=m_{\mu}^{2}\left(L_{22}+R_{22}\right) .
$$

The chargino-sneutrino diagram tends to dominate over the other diagrams in the SUSYSM contribution to $\left(g_{\mu}-2\right)$. Assuming all SUSY particle masses are the same, the SUSY-SM correction is

$$
\delta a_{\mu}^{\mathrm{SUSY}} \simeq \frac{5 \alpha_{2}^{2}+\alpha_{Y}^{2}}{48 \pi} \frac{m_{\mu}^{2}}{m_{\mathrm{SUSY}}^{2}} \tan \beta
$$

for $\tan \beta \gtrsim 1$. Here, $L_{22} \simeq R_{22}$. When the left-handed sleptons have the LFV masses between the first and second generations $\left(\left(m_{\tilde{L}}^{2}\right)_{12}\right)$, the chargino-sneutrino diagram also dominates in $\mu \rightarrow e \gamma$. In this case, $\left|R_{12}\right| \gg\left|L_{12}\right|$. Taking all SUSY-particle masses common again, we get

$$
\begin{aligned}
\operatorname{Br}(\mu \rightarrow e \gamma) & \simeq \frac{\pi}{75} \alpha\left(\alpha_{2}+\frac{5}{4} \alpha_{Y}\right)^{2}\left(G_{F}^{2} m_{\mathrm{SUSY}}^{4}\right)^{-1} \tan ^{2} \beta\left(\frac{\left(m_{\tilde{L}}^{2}\right)_{12}}{m_{\mathrm{SUSY}}^{2}}\right)^{2} \\
& =3 \times 10^{-5}\left(\frac{\delta a_{\mu}^{\mathrm{SUSY}}}{10^{-9}}\right)^{2}\left(\frac{\left(m_{\tilde{L}}^{2}\right)_{12}}{m_{\mathrm{SUSY}}^{2}}\right)^{2} .
\end{aligned}
$$

The value $\delta a_{\mu}^{\mathrm{SUSY}}>10^{-9}$ is favored by the recent result from the E821 experiment at the $2 \sigma$ level. From the current experimental bound of $\mu \rightarrow e \gamma\left(\operatorname{Br}(\mu \rightarrow e \gamma)<1.2 \times 10^{-11}\right.$ [17]), this imposes a stringent constraint on the LFV mass for the left-handed sleptons ast

$$
\frac{\left(m_{\tilde{L}}^{2}\right)_{12}}{m_{\mathrm{SUSY}}^{2}} \lesssim 6 \times 10^{-4}\left(\frac{\delta a_{\mu}^{\mathrm{SUSY}}}{10^{-9}}\right)^{-1}\left(\frac{\mathrm{Br}(\mu \rightarrow e \gamma)}{1.2 \times 10^{-11}}\right)^{\frac{1}{2}}
$$

\footnotetext{
${ }^{2}$ When only the right-handed sleptons have the LFV masses, the correlation between $\operatorname{Br}(\mu \rightarrow e \gamma)$ and $\left(g_{\mu}-2\right)$ is relatively weak. This is because the dominant contribution to $\mu \rightarrow e \gamma$ comes from bino-slepton diagrams, while bino-slepton diagrams are subdominant in $\left(g_{\mu}-2\right)$. Moreover, there is an accidental cancellation among the diagrams in $\mu \rightarrow e \gamma$ 18]. If both the left-handed and right-handed sleptons have the LFV masses, the correlation depends on the LFV masses and the other SUSY-breaking parameters. In this case, the event rate of $\mu \rightarrow e \gamma$ is determined by the competition between a neutralino-slepton diagram proportional to $m_{\tau}$ and the chargino-sneutrino diagram [19].
} 
This constraint (7) means that the off-diagonal component in the slepton mass matrix should be much smaller than the diagonal ones, so that $\mu \rightarrow e \gamma$ is suppressed. On the other hand, the LFV mass terms for sleptons are not forbidden by any symmetries in the presence of the neutrino masses. The decoupling solution (so-called effective SUSY model) has been proposed as a solution for the SUSY FCNC problem [20]. In this model the slepton and squark masses for the first and second generations are so heavy in order to suppress the FCNC processes, while the off-diagonal components in the squark and slepton mass matrixes are comparable with the diagonal ones. Now the effective SUSY model is disfavoured, since the constraint (7) is independent of the SUSY-breaking scale $m_{\text {SUSY }}$ and $\tan \beta$. The above result supports the universal scalar mass hypothesis for the SUSY breaking parameters, such as in the minimal supergravity scenario [21] or the gaugemediated SUSY-breaking scenario [22]. In this hypothesis, the charged-LFV processes are suppressed by the super-GIM mechanism. However, if the scale for the generation of the SUSY-breaking terms is higher than the right-handed neutrino mass scale in the SUSY seesaw model, the renormalization effect could induce the sizable off-diagonal components. The future charged-LFV experiments could cover $\left(m_{\tilde{L}}^{2}\right)_{12} / m_{\text {SUSY }}^{2} \gtrsim 10^{-(5-6)}$, as will be shown. Thus, these experiments will give a significant impact on the flavour models and the SUSY-breaking models.

Let us start to discuss the neutrino masses and mixing matrix in the SUSY SM with right-handed neutrinos and present the charged-LFV event rates, using the result for the neutrino oscillation experiment and $\left(g_{\mu}-2\right)$. In the SUSY SM with the right-handed neutrinos $\left(\bar{N}_{i}\right)$, the superpotential in the lepton sector is given by

$$
W=\bar{E}_{i} f_{e}^{i} L_{i} H_{d}+\bar{N}_{i} f_{\nu}^{i j} L_{j} H_{u}+\frac{1}{2} \bar{N}_{i} M_{R i} \bar{N}_{i}
$$

where $\bar{E}_{i}$ and $L_{i}$ are right-handed charged leptons and lepton doublets, respectively. Without loss of generality, we can take a basis in which the charged-lepton Yukawa coupling matrix $f_{e}$ and the right-handed neutrino mass matrix $M_{R}$ are diagonalized. In this basis the neutrino Yukawa coupling matrix $f_{\nu}$ has flavour mixings generically, and this is the origin of LFV. After integrating out the heavy right-handed neutrinos, the neutrino mass matrix is given as

$$
m_{\nu}=m_{\nu D}^{T} M_{R}^{-1} m_{\nu D}
$$


Here, the Dirac neutrino masses $m_{\nu D}$ are given by $m_{\nu D}=f_{\nu}\left\langle H_{u}\right\rangle$. The neutrino mixing matrix $V_{M N S}$ is defined by

$$
V_{M N S}^{T} m_{\nu} V_{M N S}=\operatorname{diag}\left(m_{\nu 1}, m_{\nu 2}, m_{\nu 3}\right)
$$

The tiny neutrino masses and non-zero neutrino mixings provide neutrino oscillation solutions to atmospheric and solar neutrino problems.

In order to explain the atmospheric neutrino result, we need an almost maximal mixing between $\nu_{\mu}$ and $\nu_{\tau}\left(\left(V_{M N S}\right)_{\mu 3} \simeq 1 / \sqrt{2}\right)$. Thus, the structure of the neutrino mixing matrix looks very different from that of the Kobayashi-Maskawa mixing matrix in the quark sector. So far many models have been proposed in order to naturally accommodate both small mixings in the quark sector and large mixing in the neutrino sector within the context of unified theories. An interesting proposal is that a large mixing for neutrinos can be easily understood by a lopsided structure of the Dirac mass matrices (that is, $f_{\nu}^{22} \sim f_{\nu}^{23} \ll f_{\nu}^{33} \sim f_{\nu}^{32}$ ) without introducing a non-trivial structure of the right-handed neutrino mass matrix 23]. In this case, the lepton mixing matrix $V$, which diagonalizes the neutrino Yukawa matrix as

$$
U f_{\nu} V=\operatorname{diag}\left(f_{\nu 1}, f_{\nu 2}, f_{\nu 3}\right)
$$

also has a large mixing between the second and third generations, i.e. $V_{23} \sim 1 / \sqrt{2}$. The component $V_{13}$ is model-dependent; however, it tends to be larger than 0.01 . We list typical predicted values for $V_{23}$ and $V_{13}$ in some proposed models in Table 11. The orderone $V_{23}$ and non-zero $V_{13}$ elements are very important for the charged-LFV processes in SUSY models, as stressed in Refs. [6]. The component $V_{12}$ depends on the solution of the solar neutrino problem. It may also give a sizable contribution to the LFV processes if $f_{\nu 2}$ and $V_{12}$ are relatively large [0]. However, we take a limit of vanishing $f_{\nu 2}$ in order to derive the conservative predictions in this paper.

Using the above results for the neutrino mixing matrix, we discuss the charged-LFV processes in the SUSY SM with the right-handed neutrinos. In order to describe a real world, soft SUSY-breaking terms have to be included in the SUSY SM. As mentioned above, the current situation favours the universal scalar mass hypothesis for the SUSYbreaking parameters. If the scale for the generation of the SUSY-breaking terms is higher, 


\begin{tabular}{|c|c|c|}
\hline Models & $V_{23}$ & $V_{13}$ \\
\hline Albright et al. [25] & 0.9 & 0.06 \\
Altarelli et al. [25] & 0.5 & 0.09 \\
Bando et al. 225] & $\sim 0.7$ & $\sim 0.1$ \\
Hagiwara et al. [25] & 0.7 & 0.06 \\
Nomura et al. [25] & 0.7 & $\sim 0.1$ \\
Sato et al. and Buchmüller et al. [23, 24] & 0.7 & $\sim 0.05$ \\
\hline
\end{tabular}

Table 1: Typical predicted values for $V_{23}$ and $V_{13}$ in various models [23, 24, 25].

the existence of the large neutrino Yukawa coupling can induce significant LFV masses through the renormalization effect, as pointed out in Refs. [4, 河. In the following, the scale for the generation of the SUSY-breaking terms is assumed to be the GUT scale or the reduced Planck scale, as in the minimal supergravity scenario. Since the left-handed leptons couple to the right-handed neutrinos, LFV is induced in left-handed slepton masses.

The renormalization-group equation (RGE) for the left-handed slepton masses is given by

$$
\begin{gathered}
\mu \frac{d}{d \mu}\left(m_{\tilde{L}}^{2}\right)_{i j}=\left(\mu \frac{d}{d \mu}\left(m_{\tilde{L}}^{2}\right)_{i j}\right)_{\text {SUSY-SM }} \\
+\frac{1}{16 \pi^{2}}\left\{m_{\tilde{L}}^{2} f_{\nu}^{\dagger} f_{\nu}+f_{\nu}^{\dagger} f_{\nu} m_{\tilde{L}}^{2}+2\left(f_{\nu}^{\dagger} m_{\tilde{\nu}}^{2} f_{\nu}+m_{H_{u}}^{2} f_{\nu}^{\dagger} f_{\nu}+A_{\nu}^{\dagger} A_{\nu}\right)\right\}_{i j},
\end{gathered}
$$

where $m_{\tilde{L}}, m_{\tilde{\nu}}, m_{H_{u}}$, and $A_{\nu}$ denote the SUSY-breaking masses for the doublet slepton $(\tilde{L})$, the right-handed sneutrino $(\tilde{\nu})$, the Higgs $\left(H_{u}\right)$, and the trilinear A-term for sneutrinos, respectively. Here, $\left(\mu \frac{d}{d \mu}\left(m_{\tilde{L}}^{2}\right)_{i j}\right)_{\text {SUSY-SM }}$ represents the RGE in the SUSY SM:

$$
\begin{aligned}
\left(\mu \frac{d}{d \mu}\left(m_{\tilde{L}}^{2}\right)_{i j}\right)_{\text {SUSY-SM }}= & \frac{1}{16 \pi^{2}}\left\{-\left(\frac{6}{5} g_{1}^{2} M_{1}^{2}+6 g_{2}^{2} M_{2}^{2}\right) \delta_{i j}\right. \\
& +\left(m_{\tilde{L}}^{2} f_{e}^{\dagger} f_{e}+f_{e}^{\dagger} f_{e} m_{\tilde{L}}^{2}\right)_{i j} \\
& \left.+2\left(f_{e}^{\dagger} m_{\tilde{e}}^{2} f_{e}+m_{H_{d}}^{2} f_{e}^{\dagger} f_{e}+A_{e}^{\dagger} A_{e}\right)_{i j}\right\},
\end{aligned}
$$

where $M_{1}, M_{2}, m_{\tilde{e}}, m_{H_{d}}$ and $A_{e}$ denote the bino mass, wino mass, SUSY-breaking masses for the charged slepton $(\tilde{e})$, the Higgs $\left(H_{d}\right)$, and the trilinear A-term for charged sleptons, respectively.

If we assume the universal scalar mass $\left(m_{0}\right)$ for all scalar bosons and the universal A-term $\left(A_{f}=a_{0} m_{0} f_{f}\right)$ at the unification scale $\left(M_{G}=2 \times 10^{16} \mathrm{GeV}\right)$, the only source 
of LFV is the neutrino Yukawa coupling $f_{\nu}$. An iteration gives an approximate solution to Eq. (12) for the diagonal and off-diagonal components of the left-handed slepton mass matrix:

$$
\begin{aligned}
& \left(m_{\tilde{L}}^{2}\right)_{i i} \simeq m_{0}^{2}+\frac{1}{16 \pi^{2}}\left\{\frac{36 g_{G}^{2} M_{0}^{2}}{5}-m_{0}^{2}\left(6+a_{0}^{2}\right)\left|V_{i 3} f_{\nu 3}\right|^{2}\right\} \log \frac{M_{G}}{M_{R}} \\
& \left(m_{\tilde{L}}^{2}\right)_{i j} \simeq-\frac{\left(6+a_{0}^{2}\right) m_{0}^{2}}{16 \pi^{2}} V_{i 3} V_{j 3}^{*}\left|f_{\nu 3}\right|^{2} \log \frac{M_{G}}{M_{R}}, \quad \text { for } i \neq j .
\end{aligned}
$$

Here we also assumed the gaugino mass unification $\left(M_{0}\right)$ at $M_{G}$, and the hierarchy $f_{\nu 3} \gg$ $f_{\nu 2} \gg f_{\nu 1}$. In this approximation, we neglected all charged-lepton Yukawa couplings $f_{e}$. As can be seen in Eqs. (14), the large neutrino Yukawa couplings $f_{\nu 3}$ and mixing $V$ induce large LFV masses as well as non-degeneracy in diagonal components of the slepton mass matrix. If $f_{\nu 3}=f_{\text {top }}$ at the GUT scale and $m_{0} \sim M_{0},\left(m_{\tilde{L}}^{2}\right)_{12} / m_{\mathrm{SUSY}}^{2} \sim 10^{-1} \times V_{23} V_{13}$. From Eq. (7), it is found that the models with $V_{23} \simeq 1 / \sqrt{2}$ and $V_{13} \gtrsim 0.01$ are marginal. When $m_{0}$ is much smaller than $M_{0}$, the LFV masses $\left(m_{\tilde{L}}^{2}\right)_{i j}(i \neq j)$ are suppressed with respect to the diagonal elements $\left(m_{\tilde{L}}^{2}\right)_{i i}$, and all the diagonal elements tend to be degenerate. In the limit of $m_{0}=0$ (the no-scale limit), the LFV masses are generated only at higher order. In these cases the charged-LFV processes are suppressed while the correction to the $\left(g_{\mu}-2\right)$ is not suppressed. However, the large neutrino Yukawa coupling still induces the significant effect, even if we take $m_{0}=0$ at $M_{G}$, as will be shown.

As listed in Table 1, many models for realistic fermion masses imply $V_{23} \simeq 1 / \sqrt{2}$ and $V_{13}>0.01$. Therefore, we first fix $V_{23}=1 / \sqrt{2}$ and $V_{13}=0.01$, and show the predicted $\operatorname{Br}(\mu \rightarrow e \gamma)$ and $\delta a_{\mu}^{\mathrm{SUSY}}$ in Fig. 11. Here, unification between the top-quark and tauneutrino Yukawa coupling constants $\left(f_{\nu 3}=f_{\text {top }}\right)$ at the GUT scale is assumed. We take $\tan \beta=10, M_{2}=250 \mathrm{GeV}, m_{t}=175 \mathrm{GeV}$ and $m_{\nu_{\tau}}=0.055 \mathrm{eV}$. In this case the tauneutrino Yukawa coupling $f_{\nu 3}$ at the GUT scale is 0.58 and $M_{R}$ is $2 \times 10^{14} \mathrm{GeV}$ and. In our analysis, we numerically solve the RGEs for couplings and SUSY-breaking parameters, and calculate the branching ratios of $\mu \rightarrow e \gamma$ and $\left(g_{\mu}-2\right)$ by using the complete formula given in Ref. [5].

The solid line is for a case where the scale for generation of the SUSY-breaking terms in the SUSY SM $\left(M_{X}\right)$ is the GUT scale. From this figure, a smaller $m_{0}$ region is favored to explain the recent $\left(g_{\mu}-2\right)$ result given in Eq. (1). When $m_{0}$ is larger, both $\delta a_{\mu}^{\mathrm{SUSY}}$ 
and $\operatorname{Br}(\mu \rightarrow e \gamma)$ are suppressed as in Eqs. (5) and (6). On the other hand, in the region with $m_{0} \ll M_{2}$, only $\operatorname{Br}(\mu \rightarrow e \gamma)$ is suppressed, as explained above. However, the large neutrino Yukawa coupling still induces a sizable effect on $\operatorname{Br}(\mu \rightarrow e \gamma)$, even in the no-scale limit $\left(m_{0}=0\right)$.

So far we assumed that the SUSY-breaking terms are generated at the GUT scale. In fact, this leads to the conservative bound on the $\mu \rightarrow e \gamma$ event rate. If $M_{X}$ is larger than the GUT scale, the running effect above this scale also induces LFV masses in general. Therefore, the branching ratios for the charged-LFV processes will become much larger, and our result for $M_{X}=M_{G}$ is conservative. In Fig. 1, the dashed line is for the case where $M_{X}$ is the reduced Planck scale $\left(M_{P l}=2.4 \times 10^{18} \mathrm{GeV}\right)$. Here, we use the RGE of the SUSY SM with the right-handed neutrino even above the GUT scale, while we assume the GUT relation of the gaugino masses for simplicity. Since the ratio of $M_{X}$ to $M_{R}$ is larger, the event rate becomes larger.

In order to obtain a conservative lower bound on $\operatorname{Br}(\mu \rightarrow e \gamma)$ below, we take the no-scale-type initial condition $m_{0}=0$ at $M_{G}$, and show $\operatorname{Br}(\mu \rightarrow e \gamma)$ and $\delta a_{\mu}^{\text {SUSY }}$ as a function of the left-handed smuon mass and $\tan \beta$ in Fig. 2. Here we take $V_{13}=0.01$ and $V_{23}=1 / \sqrt{2}$. As the SUSY contribution to $\left(g_{\mu}-2\right)$ increases, the branching ratio of $\mu \rightarrow e \gamma$ gets larger. In the parameter region with $\delta a_{\mu}^{\mathrm{SUSY}}>10^{-9}$, which is favored by the recent result at the $2 \sigma$ level, $\operatorname{Br}(\mu \rightarrow e \gamma)$ is larger than $10^{-14}$. This interesting region will be covered by a near-future $\mu \rightarrow e \gamma$ experiment at PSI [28 if their proposed goal of $10^{-14}$ is achieved.

In Fig. 3, we show $\operatorname{Br}(\mu \rightarrow e \gamma)$ as a function of $V_{13} V_{23}$ and the left-handed smuon mass or $\delta a_{\mu}^{\text {SUSY }}$ assuming $\tan \beta=10$. At present the experimental limit $\operatorname{Br}(\mu \rightarrow e \gamma)<$ $1.2 \times 10^{-11}$ is not very significant. However, the future expected reach at PSI $(\operatorname{Br}(\mu \rightarrow$ $e \gamma) \sim 10^{-14}$ ) will be quite significant. Since, from Fig. 2, the region favored by the present $\left(g_{\mu}-2\right)$ experiment is about $m_{\tilde{L}}<360 \mathrm{GeV}$ for $\delta a_{\mu}^{\mathrm{SUSY}}<10^{-9}$, so the future limit on

\footnotetext{
${ }^{3}$ If we seriously take the no-scale-type initial condition at the GUT scale, the slepton may be the LSP, and hence we may need a lighter SUSY particle, such as the axino. Otherwise, if we take into account the running effect above the GUT scale, which is highly dependent on GUT models, this problem would be solved [26]. The detailed studies for this model, including $b \rightarrow s \gamma$, the light Higgs boson mass, and the constraint from the negative SUSY searches, are done in Refs. [27], and the analysis shows that the region with $M_{2} \lesssim 180 \mathrm{GeV}$ or $\tan \beta \lesssim(2-5)$ is excluded if the SUSY breaking terms are generated at the GUT scale.
} 
lepton mixing $V_{13}\left(\frac{V_{23}}{1 / \sqrt{2}}\right)$ will be about $4 \times 10^{-3}$. This limit will have an impact on the fermion mass models as listed in Table 1. We should stress again that in Figs. 2 and 3 , we took the no-scale-type initial condition $\left(m_{0}=0\right)$ at the GUT scale, which significantly suppress the flavour violation in the SUSY-breaking parameters as can be seen in Fig. 11. So if we consider non-zero universal mass $\left(m_{0} \neq 0\right)$ or if $M_{X}$ is larger than $M_{G}$, the branching ratio of $\mu \rightarrow e \gamma$ becomes larger.

Finally, we comment on another interesting process, the $\mu-e$ conversion in nuclei. In the SUSY see-saw model, the photon penguin diagram tends to dominate over the other contributions in the $\mu-e$ conversion process in a wide range of the parameter space. Therefore, we have the following relation between event rates $\operatorname{Br}(\mu \rightarrow e \gamma)$ and $\mathrm{R}(\mu \rightarrow e$ in nuclei):

$$
\frac{\mathrm{R}(\mu \rightarrow e \text { in } \mathrm{Ti}(\mathrm{Al}))}{\operatorname{Br}(\mu \rightarrow e \gamma)} \simeq 5(3) \times 10^{-3}
$$

The proposed experiment MECO at BNL will reach $10^{-16}$ for the conversion rate in Al [29]. From relation (15), it is found that the MECO experiment will also provide a significant probe on LFV in the SUSY SM. Furthermore, according to ongoing studies for the $\nu$ factory at CERN [30] and the PRISM project at KEK/JAERI [31], a sensitivity of $10^{-18}$ for $\mathrm{R}(\mu \rightarrow e$ in $\mathrm{Ti})$ may be possible. A value of $\mathrm{R}(\mu \rightarrow e$ in $\mathrm{Ti}) \lesssim 10^{-18}$ corresponds

to $\operatorname{Br}(\mu \rightarrow e \gamma) \lesssim 10^{-16}$, therefore $V_{13} \lesssim 10^{-(3-4)}$. If this is achieved and the anomaly of $\left(g_{\mu}-2\right)$ is also well-established in the future, not only realistic fermion-mass models but also the SUSY-breaking mechanism under the universal scalar mass hypothesis, including no-scale-type (or gaugino-mediation) SUSY breaking, will be significantly probed by the LFV and $\left(g_{\mu}-2\right)$ experiments.

\section{Acknowledgement}

This work was also supported in part by the Grant-in-Aid for Scientific Research from the Ministry of Education, Science, Sports and Culture of Japan, on Priority Area 707 "Supersymmetry and Unified Theory of Elementary Particles" (J.H.).

\section{References}


[1] Y. Fukuda et al. [Super-Kamiokande Collaboration], Phys. Rev. Lett. 81, 1562 (1998) hep-ex/9807003; ibid 85, 3999 (2000) hep-ex/0009001.

[2] R. J. Davis, D. S. Harmer and K. C. Hoffman, Phys. Rev. Lett. 20, 1205 (1968);

Y. Fukuda et al. [Kamiokande Collaboration], Phys. Rev. Lett. 77, 1683 (1996);

K. Lande et al., Astrophys. J. 496, 505 (1998);

D. N. Abdurashitov et al., Phys. Lett. B 328, 234 (1994);

P. Anselmann et al. [GALLEX Collaboration.], Phys. Lett. B 342, 440 (1995);

Y. Fukuda et al. [Super-Kamiokande Collaboration], Phys. Rev. Lett. 82, 1810 (1999).

[3] T. Yanagida, in Proceedings of the Workshop on Unified Theory and Baryon Number of the Universe, Tsukuba, Japan, 1979, edited by O. Sawada and A. Sugamoto (KEK, Tsukuba, 1979);

M. Gell-Mann, P. Ramond, and R. Slansky, in Supergravity, Proceedings of the Workshop, Stony Brook, NY, 1979, edited by P. van Nieuwenhuizen and D. Freedman (North-Holland, Amsterdam, 1979).

[4] F. Borzumati and A. Masiero, Phys. Rev. Lett. 57, 961 (1986);

J. Hisano, T. Moroi, K. Tobe, M. Yamaguchi and T. Yanagida, Phys. Lett. B 357, 579 (1995) hep-ph/9501407.

[5] J. Hisano, T. Moroi, K. Tobe and M. Yamaguchi, Phys. Rev. D 53, 2442 (1996) hep-ph/9510309.

[6] J. Sato, K. Tobe and T. Yanagida, Phys. Lett. B 498, 189 (2001) hep-ph/0010348;

J. Sato and K. Tobe, hep-ph/0012333.

[7] J. Hisano and D. Nomura, Phys. Rev. D 59, 116005 (1999) hep-ph/9810479 :hepph/0004061.

[8] J. Hisano, D. Nomura and T. Yanagida, Phys. Lett. B 437, 351 (1998) hepph/9711348];

J. Ellis, M. E. Gomez, G. K. Leontaris, S. Lola and D. V. Nanopoulos, Eur. Phys. J. C 14, 319 (2000) hep-ph/9911459;

J. L. Feng, Y. Nir and Y. Shadmi, Phys. Rev. D 61, 113005 (2000) hep-ph/9911370; 
W. Buchmüller, D. Delepine and L. T. Handoko, Nucl. Phys. B 576, 445 (2000) hepph/9912317.

[9] H. N. Brown et al. [Muon g-2 Collaboration], hep-ex/0102017.

[10] A. Czarnecki and W. J. Marciano, hep-ph/0102122.

[11] R. Barbieri and L. Maiani, Phys. Lett. B 117, 203 (1982);

D. A. Kosower, L. M. Krauss and N. Sakai, Phys. Lett. B 133, 305 (1983);

T. C. Yuan, R. Arnowitt, A. H. Chamseddine and P. Nath, Z. Phys. C 26, 407 (1984);

C. Arzt, M. B. Einhorn and J. Wudka, Phys. Rev. D 49, 1370 (1994) hepph/9304206];

J. L. Lopez, D. V. Nanopoulos and X. Wang, Phys. Rev. D 49, 366 (1994) hepph/9308336; ;

U. Chattopadhyay and P. Nath, Phys. Rev. D 53, 1648 (1996) hep-ph/9507386;

T. Moroi, Phys. Rev. D 53, 6565 (1996) [hep-ph/9512396;

M. Carena, G. F. Giudice and C. E. Wagner, Phys. Lett. B 390, 234 (1997) hep$\mathrm{ph} / 9610233$.

[12] L. Everett, G. L. Kane, S. Rigolin and L. Wang, hep-ph/0102145;

J. L. Feng and K. T. Matchev, hep-ph/0102146;

E.A. Baltz and P. Gondolo, hep-ph/0102147;

U. Chattopadhyay and P. Nath, hep-ph/0102157;

S. Komine, T. Moroi and M. Yamaguchi, hep-ph/0102204.

[13] E. Ma and M. Raidal, hep-ph/0102255.

[14] T. Huang, Z. H. Lin, L. Y. Shan and X. Zhang, hep-ph/0102193.

[15] J. Ellis, C. Kounnas and D. V. Nanopoulos, Nucl. Phys. B 247, 373 (1984);

A. B. Lahanas and D. V. Nanopoulos, Phys. Rep. 145, 1 (1987).

[16] D. E. Kaplan, G. D. Kribs and M. Schmaltz, Phys. Rev. D 62, 035010 (2000) hepph/9911293; ;

Z. Chacko, M. A. Luty, A. E. Nelson and E. Ponton, JHEP 0001, 003 (2000);

M. Schmaltz and W. Skiba, Phys. Rev. D 62, 095005 (2000) hep-ph/0001172. 
[17] M. L. Brooks et al. [MEGA Collaboration], Phys. Rev. Lett. 83, 1521 (1999) hepex/9905013.

[18] J. Hisano, T. Moroi, K. Tobe and M. Yamaguchi, Phys. Lett. B 391, 341 (1997) hep-ph/9605296.

[19] Y. Okada, K. Okumura and Y. Shimizu, Phys. Rev. D 61, 094001 (2000) hepph/9906446].

[20] M. Dine, A. Kagan and S. Samuel, Phys. Lett. B 243, 250 (1990);

S. Dimopoulos and G. F. Giudice, Phys. Lett. B 357, 573 (1995) hep-ph/9507282;

A. Pomarol and D. Tommasini, Nucl. Phys. B 466, 3 (1996) hep-ph/9507462;

A. G. Cohen, D. B. Kaplan and A. E. Nelson, Phys. Lett. B 388, 588 (1996) hepph/9607394];

J. Hisano, K. Kurosawa and Y. Nomura, Phys. Lett. B 445, 316 (1999) hepph/9810411; Nucl. Phys. B 584, 3 (2000) hep-ph/0002286.

[21] For a review, see H. P. Nilles, Phys. Rep. 110, 1 (1984).

[22] M. Dine, A. E. Nelson, Y. Nir and Y. Shirman, Phys. Rev. D 53, 2658 (1996) hepph/9507378];

for a review, see G. F. Giudice and R. Rattazzi, Phys. Rep. 322, 419 (1999) hep$\mathrm{ph} / 9801271]$.

[23] J. Sato and T. Yanagida, Phys. Lett. B 430, 127 (1998);

C. H. Albright, K. S. Babu and S. M. Barr, Phys. Rev. Lett. 81, 1167 (1998) hepph/9802314];

N. Irges, S. Lavignac and P. Ramond, Phys. Rev. D 58, 035003 (1998) hepph/9802334];

W. Buchmüller and T. Yanagida, Phys. Lett. B 445, 399 (1999) hep-ph/9810308.

[24] J. Sato and T. Yanagida, hep-ph/0009205.

[25] For examples, C. H. Albright and S. M. Barr, Phys. Rev. D 62, 093008 (2000) hepph/0003251];

K. Hagiwara and N. Okamura, Nucl. Phys. B 548, 60 (1999) hep-ph/9811495;

M. Bando, T. Kugo and K. Yoshioka, Phys. Lett. B 483, 163 (2000) |hep- 
ph/0003231];

G. Altarelli, F. Feruglio and I. Masina, hep-ph/0007254;

Y. Nomura and T. Yanagida, Phys. Rev. D 59, 017303 (1999) hep-ph/9807325].

[26] S. Komine and M. Yamaguchi, Phys. Rev. D 63, 035005 (2001) hep-ph/0007327.

[27] S. Komine, hep-ph/0102030;

S. Komine, T. Moroi and M. Yamaguchi, hep-ph/0103182.

[28] L.M. Barkov et al., Research Proposal to PSI, 1999. See also http://www.icepp.s.utokyo.ac.jp/meg/.

[29] M. Bachman et al. [MECO Collaboration], Proposal to BNL, 1997. See also http://meco.ps.uci.edu.

[30] See the WEB page of "Neutrino factory and muon storage rings at CERN"; http://muonstoragerings.web.cern.ch/muonstoragerings/.

[31] See technical notes in the homepage of the PRISM project: http://www-prism.kek.jp. 


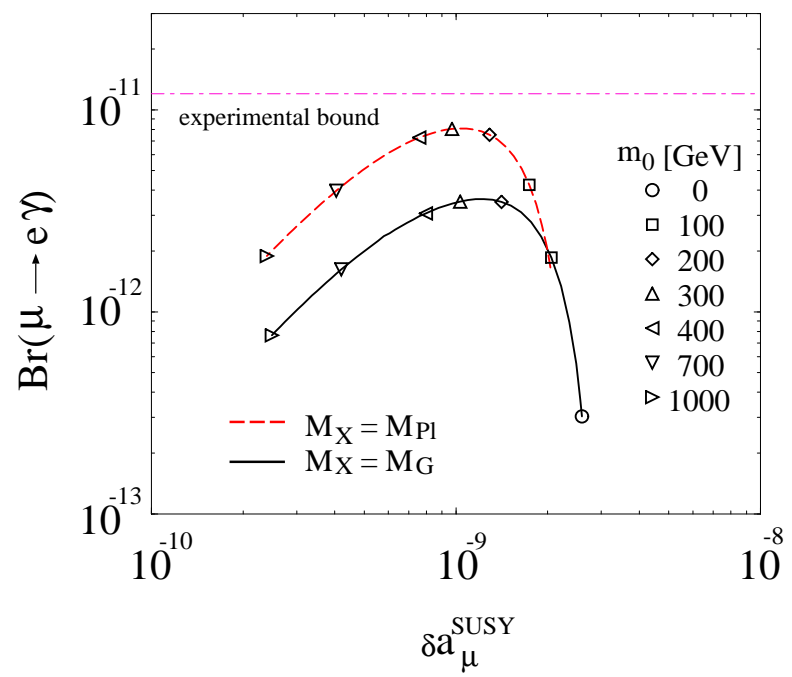

Figure 1: $\operatorname{Br}(\mu \rightarrow e \gamma)$ and $\delta a_{\mu}^{\mathrm{SUSY}}$ as a function of universal mass $m_{0}$. Here we take $V_{13}=0.01, V_{23}=1 / \sqrt{2}, \tan \beta=10$ and $M_{2}=250 \mathrm{GeV}$. We assume unification between the top-quark and tau-neutrino Yukawa couplings $\left(m_{t}=175 \mathrm{GeV}\right.$ and $\left.m_{\nu_{\tau}}=0.055 \mathrm{eV}\right)$. The solid and dashed lines are for cases where the scale for the generation of the SUSYbreaking terms in the SUSY SM $\left(M_{X}\right)$ are the GUT scale and the reduced Planck scale, respectively. 


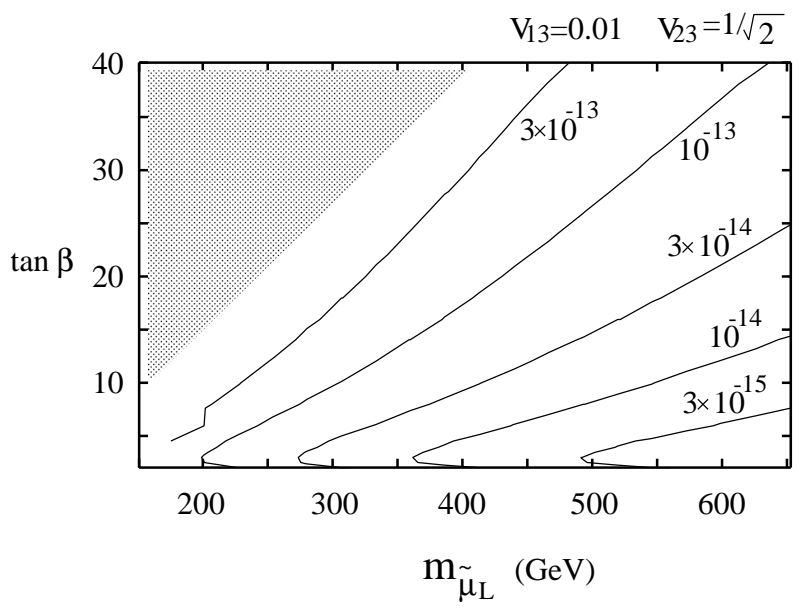

(a)

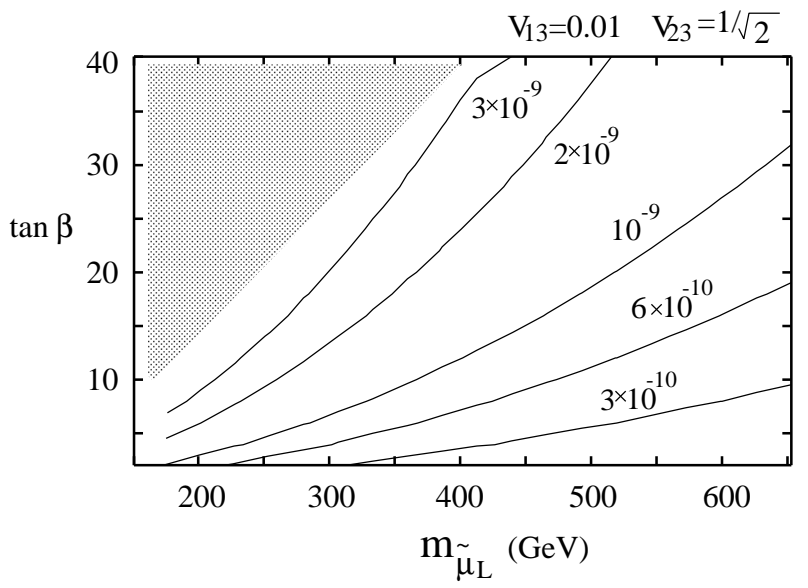

(b)

Figure 2: (a) $\operatorname{Br}(\mu \rightarrow e \gamma)$ and (b) $\delta a_{\mu}^{\text {SUSY }}$ as functions of the left-handed smuon mass and $\tan \beta$. Here, we impose the no-scale condition $\left(m_{0}=0\right)$ at the GUT scale in order to derive the conservative lower bound on $\operatorname{Br}(\mu \rightarrow e \gamma)$. We take $V_{13}=0.01$ and $V_{23}=1 / \sqrt{2}$, and assume unification between the top-quark and tau-neutrino Yukawa couplings as in Fig. 11. 


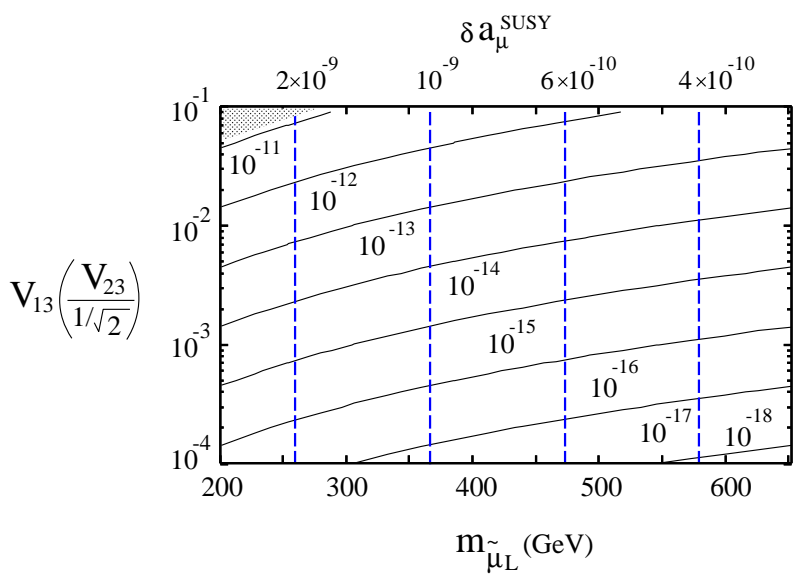

Figure 3: $\operatorname{Br}(\mu \rightarrow e \gamma)$ as a function of $V_{13} V_{23}$ and the left-handed smuon mass or $\delta a_{\mu}^{\mathrm{SUSY}}$ assuming $\tan \beta=10$. We impose the no-scale condition $\left(m_{0}=0\right)$ at the GUT scale. Unification between the top-quark and tau-neutrino Yukawa couplings is assumed as in Fig. 1. In typical models, $V_{13} \gtrsim 10^{-2}$, as listed in Table 1. 